\section{Acute disseminated encephalomyelitis after parenteral therapy with herbal extracts: a report of two cases}

Stefan Schwarz, Michael Knauth, Stefan Schwab, Ingeborg Walter-Sack,
Eckhard Bonmann, Brigitte Storch-Hagenlocher

Department of

Neurology, University of Heidelberg, $400 \mathrm{Im}$ Neuenheimer Feld,

Heidelberg 69120,

Germany

S Schwarz

S Schwab

E Bonmann

B Storch-Hagenlocher

Department of

Neuroradiology

M Knauth

Department of Clinical

Pharmacology and

Pharmacoepidemiology,

Department of

Medicine, University

of Heidelberg, 58

Bergheimer Strasse,

Heidelberg 69115,

Germany

I Walter-Sack

Correspondence to:

Dr Stefan Schwarz

stefan_schwarz@med.

uni-heidelberg.de

Received 1 March 2000

and in revised form

26 May 2000

Accepted 6 June 2000

Abstract

Two patients with acute disseminated encephalomyelitis after repeated injection of extracts from several different plants are described. There was no evidence of prior infection or vaccination. Both patients recovered rapidly after treatment with methylprednisolone. Acute disseminated encephalomyelitis should be considered a rare complication of parenteral therapy with herbal extracts.

(F Neurol Neurosurg Psychiatry 2000;69:516-518)

Keywords: acute disseminated encephalomyelitis; phytotherapy; adverse effect

Acute disseminated encephalomyelitis $(\mathrm{ADEM})$ is an unusual monophasic or multiphasic demyelinating disorder of the CNS. Although the pathophysiology of ADEM is largely unknown, an autoimmune response to myelin basic protein triggered by infection or immunisation is strongly suspected to be the main aetiological factor. ${ }^{12}$ The disease typically occurs after infections or vaccinations. ${ }^{23}$ However, in many patients with ADEM, no evidence of prior infection or vaccination can be found.
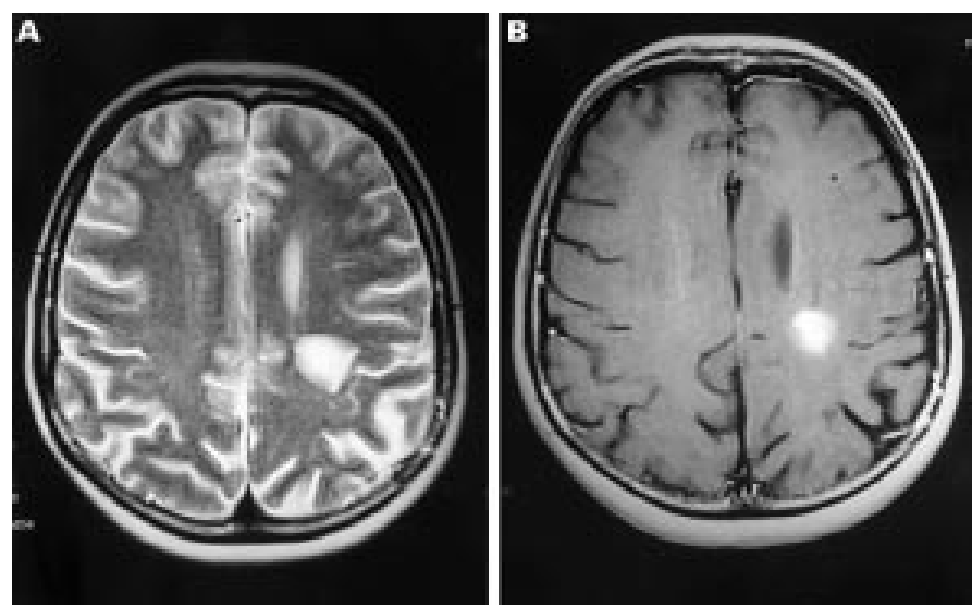

Figure 1 Patient 1. (A) Cranial T2 weighted MRI shows a large hyperintense lesion in the parietal paraventricular white matter. (B) After administration of gadolinium, the T1 weighted sequences show homogeneous enhancement (the second, smaller lesion is not visible on these sections).
We report on two patients with ADEM after repeated injection of herbal extracts.

\section{Case reports}

CASE 1

A 49 year old woman without previous neurological disease presented in January 1999 with a 5 day history of progressive numbness and weakness in the right arm. For the last 7 weeks before presentation, her familiy doctor had given an intramuscular injection of $2 \mathrm{ml}$ of a plant extract (Echinacea angustifolia D2 1.1 $\mathrm{ml}$, aconitum D4 $0.3 \mathrm{ml}$, and lachesis D8 (a snake venom) $0.3 \mathrm{ml}$; "Echinacea comp. Hevert inject ${ }^{\mathbb{}}$ ", Hevert-Arzneimittel, Nussbaum, Germany), mixed with $5 \mathrm{ml}$ of her venous blood twice a week. The last of these treatments was given 14 days before the onset of neurological symptoms. The aim of this therapy was to prevent viral infections and to prophylactically "boost the immune system". Except for chronic back pain, the patient had been otherwise well, and there was no history of infection, fever, or vaccination in the weeks before presentation. Her family history was negative for multiple sclerosis.

On admission, the patient exhibited mild spastic paresis and fluctuating numbness of the right arm. The CSF contained $0.24 \mathrm{~g}$ protein $/ 1$ and 11 leucocytes $/ \mu$ ( $>90 \%$ lymphocytes). Immunoglobulin analysis of CSF and serum showed a slightly increased CSF IgG (47 mg/1). Oligoclonal bands were present in the CSF only. Other routine laboratory indices as well as serological markers for other autoimmune diseases were all normal. An extensive microbiological investigation, including CSF markers litis, showed no evidence of recent infection. Cranial T2 weighted MRI and FLAIR sequences showed two distinct hyperintense lesions in the left parietal paraventricular deep white matter, both homogeneously enhancing after gadolinium in the $\mathrm{T} 1$ weighted sequences (fig $1 \mathrm{~A}$ and $\mathrm{B}$ ). Visual evoked potentials (VEPs) were normal on both sides.

The clinical course was favourable. After infusion of $500 \mathrm{mg}$ methylprednisolone daily over 5 days, the symptoms improved rapidly. Seven months after presentation, the patient had minimal residual coordination difficulties for viral and bacterial agents causing encepha- 

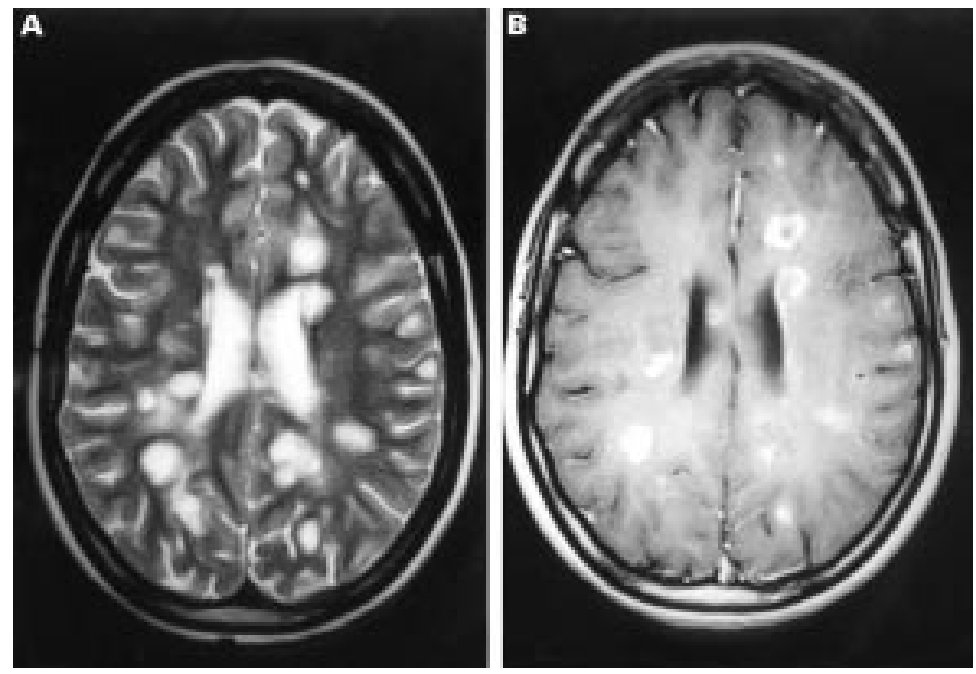

Figure 2 Patient 2. (A) Cranial T2 weighted MRI shows multiple hyperintense lesions of varying size in the subcortical and periventricular white matter of both hemispheres. (B) Gadolinium enhanced $T 1$ weighted sequences show enhancement in all lesions.

in the right hand. In the interim, the patient had been well and had not experienced any new neurological symptom. Cranial MRI was repeated and showed a residual paraventricular, left parietal small hyperintense lesion on the FLAIR and T2 weighted sequences.

CASE 2

A 23 year old woman was admitted in February 1998 with a 14 day history of progressive memory dysfunction and psychomotor retardation. Weakness of the right arm had been noted for 4 days. The patient had been well until the onset of symptoms, and she had no history of neurological symptoms or of a recent infection, vaccination, fever, or arthritis. Her family history was negative for multiple sclerosis. As an alternative treatment for being overweight, the patient had been treated for 4 weeks with subcutaneous injections of a plant extract in a private clinic specialising in obesity. The preparation consisted of a saline solution of Adonis vernalis D6, calcium carbonicum D14, capsicum D8, Cascara sagrada D6, Fucus vesiculosus D6, graphites D14, phylolacca D5, and sulfur D12. In the first 2 weeks, the patient received one subcutaneous injection of $1 \mathrm{ml}$ daily; in the second 2 weeks $1 \mathrm{ml}$ was administered every other day. She reported to have lost $13 \mathrm{~kg}$ in weight under this regimen, which was accompanied by a balanced reduction diet.

On presentation, the patient was somnolent, and showed severe concentration, attention, and memory deficits. There was a moderate paresis in the right arm. Examination of CSF showed 11 leucocytes/ $\mu$ l (96\% lymphocytes); protein $(0.23 \mathrm{~g} / \mathrm{l})$ and $\mathrm{IgG}$ concentrations were normal. Oligoclonal bands were present in the CSF but not in the serum. Cultures of CSF and tests for bacterial and viral antibodies and antigens were negative. Routine laboratory indices, including markers for vasculitis and infection, were all normal. Cranial T2 weighted MRI showed multiple hyperintense lesions of varying size in the subcortical and periventricular white matter of both hemispheres and the cerebellum, with partly homogeneous and partly ring-like enhancement after gadolinium on the $\mathrm{T} 1$ weighted sequences (fig $2 \mathrm{~A}$ and $\mathrm{B}$ ). The VEPs were normal on both sides.

We treated the patient with methylprednisolone, beginning with $500 \mathrm{mg}$ daily intravenously for 5 days, and then continuing with oral administration, tapering the dose over 4 weeks. With this treatment, the patient's symptoms resolved, and 2 months after onset she had recovered. When last seen in November 1999, the patient was well, and a neurological examination showed normal findings. The patient reported that 4 months previously she had had double vision, which lasted over a period of 2 weeks and then disappeared without any specific treatment. Cranial MRI was repeated and showed residual nonenhancing hyperintense lesions in the white matter of the cerebrum and cerebellum on FLAIR and T2 weighted sequences. New abnormalities were not found.

\section{Discussion}

Phytomedicine has become increasingly popular, now constituting a huge and rapidly growing industry. ${ }^{4}$ Herbal medications are commonly perceived as "natural" and harmless; however, most herbal drugs are unlicensed products and have not been scientifically tested. Although serious adverse events are only seen infrequently, herbal drugs are by no means harmless, and there is a increasing body of literature demonstrating toxic, allergic, and anaphylactic reactions, interactions with other medications, or adverse effects due to contamination (for an overview, see Ernst ${ }^{5}$ ).

In the two patients presented here a causal relation between the treatment with herbal extracts and ADEM is highly probable. In both patients there was a close temporal relation between parenteral treatment and onset of neurological symptoms, which is compatible with the time interval of a few days up to 4 weeks usually found in patients with ADEM. ${ }^{36}$ Similar to immunisations, the herbal extracts were given repeatedly, which may have boosted the immune response.

Herbal extracts contain potentially immunogenic proteins, glycoproteins, and phospholipids. All these ingredients are able to provoke a response in the immune system, ranging from fever to classic anaphylactic allergic reactions as well as delayed immunological cellular reactions such as vasculitis, hepatitis, or delayed cutaneous responses. ${ }^{5}$ Potential cross reactivity between herbal extracts and brain protein could be a possible mechanism for inducing demyelination.

Many herbal medications consist of several different components, which makes it impossible to determine which component is responsible for the supposed beneficial or adverse effects. Moreover, possible interactions between different herbal extracts have only rarely been subjected to scientific evaluation. ${ }^{7}$ In addition, in patient 1 , the herbal medication was mixed with her own blood before injection. Perhaps presenting herbal and autogenous epitopes together could have enhanced the immune response. Of the different substances 
administered in our two patients, only echinacea preparations (patient 1) have been the subject of larger observational and a few randomised studies. Echinacea extracts are one of the most often used herbal drugs in western countries, given as prophylaxis and therapy for various bacterial, fungal, or viral infections. ${ }^{8}$ Various immunostimulant properties of echinacea extracts have been repeatedly shown both in vitro and in vivo. ${ }^{9} 10$ Whereas the efficacy of echinacea has not convincingly been shown in controlled studies, ${ }^{811}$ there are numerous reports of adverse immune reactions, including anaphylactic responses, urticaria, Löfgren's syndrome, vasculitis, and erythema multiforme. ${ }^{12-15}$

It is still difficult to differentiate between ADEM and the first manifestation of multiple sclerosis. ${ }^{2}$ However, in both of these patients, the clinical and radiological findings would have been unusual for the first manifestation of this disease. In patient 1 , the criteria for the diagnosis of multiple sclerosis are not met because of the monophasic, acute course of the disease. In addition, the age of onset (49 years) is uncommon for a first presentation of multiple sclerosis. Patient 2 also had an acute course of the disease with rapid recovery but had transient double vision a few months later. However, the initial presentation of this patient was atypical for a first manifestation of multiple sclerosis. The radiological examination in this patient showed large, diffuse symmetric involvement of both the subcortical and periventricular white matter of both cerebral and cerebellar hemispheres. All lesions showed contrast enhancement, which is well compatible with ADEM. ${ }^{3}{ }^{16}$ However, the differential diagnosis of ADEM and multiple sclerosis cannot be made with certainty in patient 2 . It may be that, in this patient, the first episode of multiple sclerosis was triggered by the parenteral administration of plant extracts, in analogy to acute exacerbations of multiple sclerosis which can occur in temporal relation to an infection or vaccination.
Our case reports of ADEM as a rare adverse event after parenteral therapy with herbal extracts are further arguments for the point of view that phytomedicine should be scientifically evaluated for safety and efficacy before being made available, and postmarketing surveillance studies for detecting safety problems should be mandatory just as for any other drug.

1 Pohl-Koppe A, Burchett SK, Thiele EA, et al. Myelin basic protein reactive $\mathrm{Th} 2 \mathrm{~T}$ cells are found in acute disseminated encephalomyelitis. F Neuroimmunol 1998;91:19-27.

2 Storch-Hagenlocher B, Griffin D. Acute disseminated encephalomyelitis (parainfectious and postvaccinal encephalitis). In: Hacke W, Hanley D, Bleck T, et al, eds. Neurocritical care. Berlin: Springer, 1994:493-9.

3 Murthy J, Yangala R, Meena A, et al. Acute disseminated encephalomyelitis: clinical and MRI study from south India. 7 Neurol Sci 1999;165:133-8.

4 Eisenberg M, Davis RB, Ettner SL, et al. Trends in alternative medicine use in the United States, 1990-7. Result of a follow-up national survey. FAMA 1998;280:1569-75.

5 Ernst E. Harmless herbs? A review of the literature. Am $\mathcal{F}$ Med 1998;104:170-8.

6 Lukes SA, Norman D. Computed tomography in acute disseminated encephalomyelitis. Ann Neurol 1983;13:567-72.

7 Erhard M, Kellner J, Wild J, et al. Effects of echinacea, aconitum, lachesis and apis extracts, and their combinations on phagocytosis of human granulocytes. Phytotherapy Research 1994;8:14-17.

8 Grimm W, Muller HH. A randomized controlled trial of the effect of fluid extract of Echinacea purpurea on the incidence and severity of colds and respiratory infections. Am F Med 1999;106:138-43.

9 Roesler J, Emmendorffer A, Steinmuller C, et al. Application of purified polysaccharides from cell cultures of the plant Echinacea purpurea to test subjects mediates activation of the phagocyte system. Int $\mathcal{F}$ Immunopharmacol 1991;13: the pha $931-41$.

10 Burger RA, Torres AR, Warren RP, et al. Echinacea-induced cytokine production by human macrophages. Int $\mathcal{f}$ Immunopharmacol 1997; 19:371-9.

11 Melchart D, Walther E, Linde K, et al. Echinacea root extracts for the prevention of upper respiratory tract infections: a double-blind, placebo-controlled randomized trial. Arch Fam Med 1998;7:541-5.

12 Arzneimittelkommission der deutschen Ärzteschaft. Wie verträglich sind Echinacea-haltige Präparate? Dtsch Ärztebl 1996;93:A-2723.

13 Schönhöfer PS, Werner B, Kuklinski M, et al. Ungewöhnliche Immunerkrankungen durch Pflanzenextrake (Echinacea, Mistel): Akute Sarkoidose. Arzneimitteltherapie 1999; 17:282-4.

14 Mullins RJ. Echinacea-associated anaphylaxis. Med f Aust 1998;168:170-1.

15 Immunallergische Reaktionen nach Echinacea-Extrakten (Echinacin, Esberitox N, ua). Arznei Telegramm 1991;4:39.

16 Kesselring J, Miller DH, Robb SA, et al. Acute disseminated encephalomyelitis. MRI findings and the distinction from multiple sclerosis. Brain 1990;113:291-302. 